\title{
UNSTABLE SOLUTIONS OF A CLASS OF HILL DIFFERENTIAL EQUATIONS*
}

\author{
BY \\ GABRIEL HORVAY \\ McDonnell Aircraft Corporation
}

1. Introduction. Linear differential equations with periodic coefficients play an important role in problems of engineering and physics. The best-known of these equations is Mathieu's equation. A somewhat more complicated equation is

$$
\frac{d^{2} v}{d \psi^{2}}+\left[\theta_{-2} e^{-2 i \psi}+\theta_{-1} e^{-i \psi}+\theta_{0}+\theta_{1} e^{i \psi}+\theta_{2} e^{2 i \psi}\right] v=0
$$

which reduces to Mathieu's equation for

$$
\theta_{0}^{*}=\theta_{0}, \quad \theta_{-1}^{*}=\theta_{-1}=\theta_{1}, \quad \theta_{-2}=\theta_{2}=0,
$$

where the asterisk is used to denote the conjugate complex quantity.

This paper is concerned with the determination of the solutions

$$
v(\psi)=e^{\sigma \psi} \sum_{-\infty}^{+\infty} c_{k} e^{i k \psi}
$$

of Eq. (1a) subject to the restrictions

$$
\theta_{0}^{*}=\theta_{0}, \quad \theta_{-1}^{*}=\theta_{1}, \quad \theta_{-2}^{*}=\theta_{2}
$$

and

$$
\theta_{1}=O(\mu), \quad \theta_{2}=O\left(\mu^{2}\right),
$$

where $\mu$ is a small positive quantity. It will be seen that solution of the problem involves the determination of the "characteristic exponent" $\sigma$ from the equation

$$
\sin i \pi \sigma=\sqrt{\overline{\mathcal{D}}} \sin \pi \sqrt{\theta_{0}},
$$

where $\mathcal{D}$ denotes the expansion

$$
\mathcal{D}=1+C_{\delta} \delta+C_{\epsilon} \epsilon+C_{\eta} \eta+C_{\delta^{2} \delta^{2}}+C_{\delta \epsilon} \delta \epsilon+\cdots
$$

in the three real combinations

$$
\delta=\theta_{-1} \theta_{1}, \quad \epsilon=\theta_{-2} \theta_{2}, \quad \eta=\frac{1}{2}\left(\theta_{1}^{2} \theta_{-2}+\theta_{-1}^{2} \theta_{2}\right)
$$

of the four quantities, real and imaginary parts of $\theta_{1}$ and $\theta_{2} . \mathscr{D}$ is a power series in $\mu^{2}$ since

$$
\delta=O\left(\mu^{2}\right), \quad \epsilon=O\left(\mu^{4}\right), \quad \eta=O\left(\mu^{4}\right) .
$$

The coefficients $C$ of the series depend on $\theta_{0}$ alone.

The numerical evaluation of the coefficients of the expansion is the principal aim of this paper. This is best accomplished by first re-expressing the "doubly infinite" Hill determinant $\Phi$ in terms of its "simply infinite" principal subdeterminants $D_{n}$,

* Received June 13, 1946. 


$$
\mathcal{D}=f\left(D_{0}, D_{1}, D_{2}, \cdots\right),
$$

and then expanding $D_{n}$ into the series

$$
D_{n}=1+A_{\delta}^{n} \delta+A_{\epsilon}^{n} \epsilon+A_{\eta}^{n} \eta+A_{\delta^{2} \delta}^{n}+\cdots .
$$

The coefficients of the expansions (7) and (8) are tabulated in Tables II and I respectively for a convenient range of $\theta_{\mathbf{0}}$. For the sake of simpler printing the notation

$$
A_{\delta^{i} e^{i} \eta^{k}} \equiv\left\{n, \delta^{i} \epsilon^{i} \eta^{k}\right\}
$$

will be used whenever the subscript of $A$ becomes excessively long.

The practical solution of Eq. (1) is carried out in four steps. First, the determinants $D_{n}$, Eqs. (8), are evaluated by means of Table I. Next $\mathcal{D}$, Eq. (7), is determined from Table II. The third step consists in solving Eq. (4) or one of its variants (13a, b, c) for $\sigma$, and the last step is the determination of the coefficients $c_{k}$ of solution (2). A convenient method for carrying out this last step is discussed in Section 2. The derivations of the formulas for $\left\{n, \delta^{i} \epsilon^{i} \eta^{k}\right\}$ and for the coefficients of (7) are presented in Section 3. A numerical example is given in Section 4.

The present paper is based on a study which was recently undertaken at the McDonnell Aircraft Corp. under the sponsorship of the Bureau of Aeronautics, U. S. Navy Department. The study was prompted by recent instances of control difficulties of some helicopters and rotor blade failures of others. As will be shown in a separate paper, ${ }^{1}$ the natural modes in which hinged rotor blades flap can be represented by solutions of Eq. (1) multiplied by suitable damping factors. It will be found that the stability of the blade motion decreases as the speed of advance of the helicopter increases (as $\mu$ increases). Nevertheless, instability does not set in, because an aerodynamic damping effect outweighs, at all feasable speeds, the tendency towards instability which results from the flapping motion.

The writer's thanks are due to his colleague, Elizabeth J. Spitzer, for checking the derivations and the numerical work. The writer also wishes to express his indebtedness to Messrs. W. R. Foote, H. Poritsky and J. J. Slade, who in their paper on rotational instability of shafts ${ }^{2}$ applied a Laplace expansion to a doubly infinite determinant, and thus suggested the present approach.

2. Method of solution. The solution of Eq. (1a) is assumed in the standard form

$$
v(\psi)=e^{\sigma \psi} \sum_{-\infty}^{+\infty} c_{k} e^{i k \psi}
$$

Substitution of expression (2) into Eq. (1a) leads to the infinite set of homogeneous equations for the coefficients $c_{k}(\sigma)$ :

$$
\begin{array}{lcl}
k=-2: & \theta_{2} c_{-4}+\theta_{1} c_{-3}+\left[(\sigma-2 i)^{2}+\theta_{0}\right] c_{-2}+\theta_{-1} c_{-1}+\theta_{-2} c_{0} & =0, \\
k=-1: & \theta_{2} c_{-3}+\theta_{1} c_{-2}+\left[(\sigma-i)^{2}+\theta_{0}\right] c_{-1}+\theta_{-1} c_{0}+\theta_{-2} c_{1} & =0,(9) \\
k=0: & \theta_{2} c_{-2}+\theta_{1} c_{-1}+\left[\sigma^{2}+\theta_{0}\right] c_{0}+\theta_{-1} c_{1}+\theta_{-2} c_{2} & =0,
\end{array}
$$

${ }^{1} \mathrm{G}$. Horvay, Rotor blade flapping motion, to be published soon.

2 W. R. Foote, H. Poritsky and J. J. Slade, Critical speeds of a rotor with unequal shaft flexibilities, mounted in bearings of unequal flexibility, Journal of Applied Mechanics, 10, A77, 1943. 
$k=-1$ :

$$
\begin{array}{r}
\theta_{2} c_{-1}+\theta_{1} c_{0}+\left[(\sigma+i)^{2}+\theta_{0}\right] c_{1}+\theta_{-1} c_{2}+\theta_{-2} c_{3}=0, \\
\theta_{2} c_{0}+\theta_{1} c_{1}+\left[(\sigma+2 i)^{2}+\theta_{0}\right] c_{2}+\theta_{-1} c_{3}+\theta_{-2} c_{4}=0,
\end{array}
$$$$
k=-2 \text { : }
$$

The equations are consistent if their determinant, $\Delta(\sigma)$, vanishes. The consistency criterion

$$
\Delta(\sigma)=0
$$

can be expressed in the much simpler form 8,4

where

$$
\sin i \pi \sigma= \pm \sqrt{\Phi} \sin \pi \sqrt{\theta_{0}},
$$

$$
\Phi \equiv \Delta(0)=\left|\begin{array}{ccccccc}
\cdot & \cdot & \cdot & . & . & . & \cdot \\
\cdot & 1 & \theta_{-1} y_{2} & \theta_{-2} y_{2} & 0 & 0 & . \\
\cdot & \theta_{1} y_{1} & 1 & \theta_{-1} y_{1} & \theta_{-2} y_{1} & 0 & . \\
\cdot & \theta_{2} y_{0} & \theta_{1} y_{0} & 1 & \theta_{-1} y_{0} & \theta_{-2} y_{0} & . \\
\cdot & 0 & \theta_{2} y_{1} & \theta_{1} y_{1} & 1 & \theta_{-1} y_{1} & . \\
\cdot & 0 & 0 & \theta_{2} y_{2} & \theta_{1} y_{2} & 1 & . \\
. & . & . & . & . & . & .
\end{array}\right|
$$

is the determinant of system (9) for $\sigma=0$ when each equation is divided by the coefficient of the diagonal term, and

$$
y_{k}=\frac{1}{\theta_{0}-k^{2}} .
$$

$\Phi$ is either positive or negative, and so is $\theta_{0}$. Thus the quantity $\sqrt{\Phi} \sin \pi \sqrt{\theta_{0}}$ is either real or pure imaginary. In the first case set

$$
q=\sqrt{\mathscr{D}} \sin \pi \sqrt{\theta_{0}}=-\sqrt{-\mathcal{D}} \sinh \pi \sqrt{-\theta_{0}} .
$$

In the second case set

$$
q^{\prime}=q / i=-\sqrt{-\mathcal{D}} \sin \pi \sqrt{\theta_{0}}=-\sqrt{\mathcal{D}} \sinh \pi \sqrt{-\theta_{0}} .
$$

Then the solution of the transcendental equation (4) is given by

$$
\begin{aligned}
& \pm \sigma=\frac{1}{\pi} \log \left(q / i+\sqrt{1-q^{2}}\right)+m i \quad(m=0, \pm 1, \pm 2, \cdots), \\
& =\frac{i}{\pi} \arctan \frac{-q}{\sqrt{1-q^{2}}}+m i \quad \text { for } \quad-1 \leqq q \leqq 1, \\
& =\frac{1}{\pi} \log \left(q+\sqrt{q^{2}-1}\right)+\left(m-\frac{1}{2}\right) i \text { for } q \leqq-1, q \geqq 1 \text {, } \\
& =\frac{1}{\pi} \log \left(q^{\prime}+\sqrt{q^{\prime 2}}+\overline{1}\right)+m i \quad \text { for } q \text { imaginary. }
\end{aligned}
$$

${ }^{3}$ Whittaker and Watson, $A$ course of modern analysis, Cambridge, 1927, p. 416.

4 M. J. O. Strutt, Lamésche, Mathieusche und verwondte Funktionen in Physik und Technik, Springer 1932 (Edward Bros., 1944), p. 22. 
Once $\mathscr{D}$ is known, the calculation of $\sigma$ from (13) is simple matter. For any $m$ there are two solutions $\sigma_{1}$ and $\sigma_{2}$ which differ only in sign

$$
\sigma_{2}=-\sigma_{1} \text {. }
$$

Let $\sigma_{1}$ be the solution with the positive real part

$$
\sigma_{1}=\sigma_{r}+i \sigma_{i}, \quad \sigma_{r} \geqq 0 .
$$

The function $v_{1}(\psi)$ (or $v_{2}(\psi)$ ) associated with $\sigma_{1}$ (or with $\sigma_{2}$ ) is readily obtained by placing $\sigma_{1}\left(\right.$ or $\left.\sigma_{2}\right)$ into the system (9) which is now limited to the equations $k=-N$, $-N+1, \cdots,-1,+1, \cdots,+N$. Assuming $c_{k}=0$ for $k<-N$ and $k>+N$, one can solve the $2 N$ equations for $c_{-N}, c_{-N+1}, \cdots, c_{-1}, c_{1}, \cdots, c_{N}$ in terms of the arbitrary constant $c_{0}$, and then use equation $k=0$ as a check. The greater is $N$, the more harmonics are taken into account, and the more accurate is the solution. In practice the calculations are most conveniently carried out by solving equations $k=N$ and $k=-N$ for $c_{N}$ and $c_{-N}$ in terms of the variables $c_{N-1}, c_{N-2}$ and $c_{-N+1}, c_{-N+2}$, respectively. The results are then substituted into equations $k=N-1$ and $k=-N+1$; similarly $c_{N-1}$ and $c_{-N+1}$ are determined. Continuing the process one finally arrives at equations $k=+1$ and $k=-1$ involving the two variables $c_{1}$ and $c_{-1}$ only, and the parameter $c_{0}$ which can be assumed as 1 . One eliminates one of the unknowns, say $c_{-1}$ determines from the real and imaginary parts of the remaining equation the real and imaginary parts of $c_{1}$, and then, retracing the steps, obtains in succession the numerical values of $c_{-1}, c_{2}, c_{-2}, \cdots, c_{N}, c_{-N}$.

Evidently, in principle, it is immaterial what $m i(m=0, \pm 1, \pm 2, \cdots)$ is used in the $\sigma$ of Eqs. (9). For instance, the set of equations $k=-N$ to $+N$ with $m=2$, the set of equations $k=-N-2$ to $+N-2$ with $m=4$, and the set of equations $k=-N+3$ to $+N+3$ with $m=-1$ are identical. Thus, as one passes to the limit $N \rightarrow \infty$, any $m$ and any $2 N+1$ adjoining equations will lead to the same function $v_{1}(\psi)$ [or $v_{2}(\psi)$ ]. In practice, where one is limited to a finite number of equations, $2 N+1$, it is best to use the centrally located equations $k=-N$ to $+N$ with an $m$ which makes $c_{0}$ the dominating term in the series (2).

In general $v_{1}$, associated with $\sigma_{1}$, and $v_{2}$, associated with $\sigma_{2}$, are linearly independent functions. An exceptional case arises when $\sigma_{r}=0$, and $\sigma_{i}$ is an integral multiple of $\frac{1}{2}$. Then substitution of $\sigma_{1}$ and $\sigma_{2}$ into the system (9) leads to the same function

$$
v_{1}=e^{i \nu \downarrow} \sum_{-\infty}^{+\infty} c_{k} e^{i k \psi}, \quad\left(\nu=0 \text { or } \frac{1}{2}\right)
$$

The second, linearly independent, solution is now a "quasiperiodic function":4a

$$
v_{2}=e^{i \nu \psi}\left[\psi \sum_{-\infty}^{+\infty} c_{k} e^{i k \psi}+\sum_{-\infty}^{+\infty} d_{k} e^{i k \psi}\right], \quad\left(\nu=0, \frac{1}{2}\right) .
$$

For convenience the functions (15a) will be called "purely periodic" functions.

Determination of the purely periodic solutions (15a) forms the subject matter of most investigations on Mathieu and Hill differential equations. The purely periodic

4a M. J. O. Strutt, loc. cit., p. 23. As an exception there may be two purely periodic solutions. For instance, for $\theta_{0}=4, \theta_{1}=\theta_{2}=0$, one obtains $\tau_{1}^{\prime}=\cos 2 \psi, v_{2}=\sin 2 \psi$. 
solutions are usually of greatest interest, because they separate the $\mu$-regions of stability $\left(\sigma_{r}=0 ; v_{1}\right.$ and $v_{2}$ are oscillatory) from the $\mu$-regions of instability $\left(\sigma_{r}>0, v_{1} \rightarrow \infty\right.$ as $\psi \rightarrow \infty)$. A purely periodic solution can be obtained, cf. Eqs. (12), (13), only when $q^{\prime}=0\left(\mathscr{D}=0\right.$, or perhaps $\left.\theta_{0}=k^{2}\right)$, or when $q= \pm 1\left(\mathcal{D}\right.$ and $\theta_{\mathrm{c}}$ are such that $\sqrt{\mathscr{D}} \sin \pi \sqrt{\theta_{0}}$ $=1$ or $\left.\sqrt{-\Phi} \sinh \pi \sqrt{-\theta_{0}}=1\right)$. In general $\theta_{0}=k^{2}$ does not provide a purely periodic function.

In the present analysis the principal interest is attached to the unstable solutions

$$
v_{1}(\psi)=e^{\sigma_{r} \psi} \sum_{-\infty}^{+\infty} c_{k} e^{i\left(\sigma_{i}+k\right) \psi}, \quad\left(\sigma_{r}>0\right)
$$

of (1) which, after multiplication by a damping factor $e^{-n \psi / 2}$, are still stable. These solutions are in the "transition region" which extends from the $\mu$-value for which $v(\psi)$ is purely periodic to the $\mu$-value for which $e^{-n \psi / 2} v(\psi)$ is purely periodic. It will be seen in Reference 1 that a rapidly advancing helicopter usually operates in the transition region.

3. Expansion of Hill's infinite determinant. It will be convenient to call the determinant $\mathscr{D}$, Eq. (11a), a doubly infinite determinant to indicate that it extends to infinity both upward and downward. Simply infinite determinant are the principal subdeterminants of $D$,

$$
B_{n}=\left|\begin{array}{ccccc}
1 & \theta_{-1} y_{n} & \theta_{-2} y_{n} & 0 & \cdot \\
\theta_{1} y_{n+1} & 1 & \theta_{-1} y_{n+1} & \theta_{-2} y_{n+1} & \cdot \\
\theta_{2} y_{n+2} & \theta_{1} y_{n+2} & 1 & \theta_{-1} y_{n+2} & \cdot \\
0 & \theta_{2} y_{n+3} & \theta_{1} y_{n+3} & 1 & \cdot \\
\cdot & \cdot & \cdot & \cdot & \cdot
\end{array}\right| ; E_{n}=\left|\begin{array}{ccccc}
\cdot & \cdot & \cdot & \cdot & \cdot \\
\cdot & 1 & \theta_{-1} y_{n+3} & \theta_{-2} y_{n+3} \\
\cdot & \theta_{1} y_{n+2} & 1 & \theta_{-1} y_{n+2} & \theta_{-1} y_{n+2} \\
\cdot & \theta_{2} y_{n+1} & \theta_{1} y_{n+1} & 1 & \theta_{-1} y_{n+1} \\
\cdot & 0 & \theta_{2} y_{n} & \theta_{1} y_{n} & 1
\end{array}\right| .
$$

The first extends to infinity downward, the second upward. Simply infinite determinants are also the auxiliary subdeterminants

$$
S_{n}=\left|\begin{array}{ccccc}
\theta_{1} y_{n} & \theta_{-1} y_{n} & \theta_{-2} y_{n} & 0 \\
\theta_{2} y_{n+1} & 1 & \theta_{-1} y_{n+1} & \theta_{-2} y_{n+1} \\
0 & \theta_{1} y_{n+2} & 1 & \theta_{-1} y_{n+2} & \cdot \\
0 & \theta_{2} y_{n+3} & \theta_{1} y_{n+3} & 1 & \cdot \\
\cdot & \cdot & \cdot & \cdot & \cdot
\end{array}\right| ; T_{n}=\left|\begin{array}{ccccc}
\cdot & \cdot & \cdot & \cdot & . \\
\cdot & 1 & \theta_{-1} y_{n+3} & \theta_{-2} y_{n+3} & 0 \\
\cdot & \theta_{1} y_{n+2} & 1 & \theta_{-1} y_{n+2} & 0 \\
\cdot & \theta_{2} y_{n+1} & \theta_{1} y_{n+1} & 1 & \theta_{-2} y_{n+1} \\
\cdot & 0 & \theta_{2} y_{n} & \theta_{1} y_{n} & \theta_{-1} y_{n}
\end{array}\right| .
$$

$S_{n}$ differs from $D_{n}$ only in the first column; $T_{n}$ differs from $E_{n}$ only in the rightmost column.

One readily establishes the recurrence relations

$$
\begin{aligned}
D_{n}= & D_{n+1}-\theta_{-1} y_{n} S_{n+1}+\theta_{1} \theta_{-2} y_{n} y_{n+1} S_{n+2}-\epsilon y_{n} y_{n+2} D_{n+3} \\
& +\epsilon^{2} y_{n} y_{n+1} y_{n+2} y_{n+3} D_{n+4} \\
S_{n}= & \theta_{1} y_{n} D_{n+1}-\theta_{-1} \theta_{2} y_{n} y_{n+1} D_{n+2}+\epsilon y_{n} y_{n+1} S_{n+2} .
\end{aligned}
$$

A Laplace expansion of the doubly infinite determinant $\mathcal{D}$ along a dividing line between row $k=0$ and $k=-1$ leads to the following expression involving only simply infinite determinants of type $D_{n}, E_{n}, S_{r}, T_{n}$ :

$$
\begin{aligned}
\mathscr{D}= & D_{0} E_{1}-S_{0} T_{1}+y_{0} y_{1}\left(\theta_{-1} \theta_{2} D_{1} T_{2}+\theta_{1} \theta_{-2} S_{1} E_{2}\right) \\
& -\epsilon\left(y_{0} y_{2} D_{1} E_{3}+y_{1}^{2} D_{2} E_{2}+y_{0} y_{1} S_{1} T_{2}\right) \\
& +\epsilon^{2}\left(y_{0} y_{1} y_{2} y_{3} D_{1} E_{4}+y_{0} y_{1}^{2} y_{2} E_{2} D_{3}+y_{0} y_{1}^{2} y_{2} D_{2} E_{3}\right) .
\end{aligned}
$$


Since, by virtue of $(1 \mathrm{~b})$

$$
\begin{aligned}
& E_{n}=D_{n}^{*}=D_{n}, \\
& T_{n}=S_{n}^{*} \quad\left(\neq S_{n}, \text { when } \theta_{1}^{*} \neq \theta_{1}, \theta_{2}^{*} \neq \theta_{2}\right)
\end{aligned}
$$

and by virtue of (2) and (19b)

$$
S_{n} T_{m}=O\left(\mu^{2}\right),
$$

a replacement of $E_{n}$ by $D_{n}$ and a repeated insertion of (19b) into (20) gradually eliminates all but the $D_{n}$ type of determinants from the expression for $\mathcal{D}$. It is also found that $\theta_{1}, \theta_{-1}, \theta_{2}, \theta_{-2}$ appear only in the combinations $\delta, \epsilon, \eta$ given in (6a). Thus, by virtue of (6b), the expansion of $\mathscr{D}$ progresses in powers of $\mu^{2}$. Using the notation

$$
y_{0112}=y_{0} y_{1}^{2} y_{2},
$$

one finds that to $\mu^{10}$ terms

$$
\begin{aligned}
\mathscr{D}= & D_{0} D_{1}-\delta y_{01} D_{1} D_{2}-\epsilon\left(y_{02} D_{1} D_{3}+y_{11} D_{2}^{2}\right)+\eta\left(2 y_{011} D_{2}^{2}+z y_{012} D_{1} D_{3}\right) \\
& -\delta \epsilon\left(4 y_{0112} D_{2} D_{3}+2 y_{0123} D_{1} D_{4}\right)+\epsilon^{2}\left(y_{0123} D_{1} D_{4}+2 y_{0112} D_{2} D_{3}\right) \\
& +\epsilon \eta\left(4 y_{01123} D_{2} D_{4}+2 y_{0123} D_{1} D_{6}+2 y_{01122} D_{3}^{2}\right) \\
& -\delta \epsilon^{2}\left(4 y_{01123} D_{2} D_{5}+4 y_{011223} D_{3} D_{4}+2 y_{012345} D_{1} D_{6}\right)+\cdots
\end{aligned}
$$

The same process can also be carried out for the simply infinite determinant $D_{n}$. Disregarding the exceptional case $\theta_{0}=k^{2}\left(y_{k}=\infty\right)$, one finds that to $\mu^{10}$ terms

$$
\begin{aligned}
D_{0}= & D_{1}-\delta y_{01} D_{2}+\left(-\epsilon y_{02}+2 \eta y_{012}\right) D_{3}+\left(-2 \delta \epsilon+\epsilon^{2}\right) y_{0123} D_{4} \\
& +2 \epsilon \eta y_{01234} D_{5}-2 \delta \epsilon^{2} y_{012345} D_{6}+\cdots,
\end{aligned}
$$

and $D_{n}$ is obtained from $D_{0}$ by increasing the subscripts in the latter's expression by $n$.

It will be convenient to introduce at this point the notation

$$
\begin{aligned}
\sum y_{356} & =y_{356}+y_{467}+y_{678}+\cdots, \\
\sum y_{12} \sum y_{356} & =y_{12} \sum y_{356}+y_{23} \sum y_{467}+y_{34} \sum y_{578}-\cdots .
\end{aligned}
$$

Noting that

$$
\lim _{n \rightarrow \infty} D_{n}=1 \text {, }
$$

one obtains, by repeated application of (24),

$$
\begin{aligned}
D_{0} & =\left(1-\delta y_{01}\right) D_{2}+\left(-\delta y_{12}-\epsilon y_{02}+2 \eta y_{012}\right) D_{3}+\cdots \\
& =\left[1-\left(y_{01}+y_{12}\right) \delta-y_{02} \epsilon+2 y_{012} \eta\right] D_{3}+\cdots \\
& =1+A_{\delta}^{0} \delta+A_{\epsilon}^{0} \epsilon+A_{\eta}^{0} \eta+\cdots,
\end{aligned}
$$

where

$A_{\delta}^{0}=-\sum y_{01}, \quad A_{\epsilon}^{0}=-\sum y_{02}, \quad A_{\eta}^{0}=2 \sum y_{012}, \quad A_{\delta^{2}}^{0}=\sum y_{01} \sum y_{23}, \cdots$. 
The coefficient of a general term, like $\delta \epsilon^{2}$, is obtained as follows: Equation (24a) gives rise to the following symbolic products containing $\delta \epsilon^{2}$ :

$$
\begin{aligned}
& {\left[-\delta y_{01}\right]\left[-\epsilon y_{02}\right]\left[-\epsilon y_{02}\right],} \\
& {\left[-\delta y_{01}\right]\left[\epsilon^{2} y_{0123}\right],} \\
& {\left[-\epsilon y_{02}\right]\left[-2 \delta \epsilon y_{0123}\right],} \\
& {[1]\left[-2 \delta \epsilon^{2} y_{012345}\right] .}
\end{aligned}
$$

It is found that (a) contributes

$$
-\sum y_{01} \sum y_{24} \sum y_{57}-\sum y_{02} \sum y_{34} \sum y_{67}-\sum y_{02} \sum y_{36} \sum y_{67}
$$

to $A_{\delta_{\mathbf{c}^{2}}^{2}}^{0}$ (Note that no subscripts can be repeated, nor can any be skipped as one passes from one $\sum$ to the next $\sum$; furthermore (a) gives rise to three distinct summation expressions, because $y_{k, k+1}$ can appear in the first place, in the second place, and in the third place.) The relation (b) yields

which (c) yields

$$
-\sum y_{01} \sum y_{2345}-\sum y_{0123} \sum y_{45}
$$

and (d) yields

$$
2 \sum y_{02} \sum y_{3466}+2 \sum y_{0123} \sum y_{46}
$$

$$
-2 \sum y_{012345}
$$

The contributions $(28 \mathrm{a}, \mathrm{b}, \mathrm{c}, \mathrm{d})$ sum up to $\left\{0, \delta \epsilon^{2}\right\}$. By increasing the subscripts of the expressions (28) by 2 , one obtains

$$
\begin{aligned}
A_{\delta \epsilon^{2}}^{0}= & -\sum y_{23} \sum y_{46} \sum y_{79}-\sum y_{24} \sum y_{56} \sum y_{79}-\sum y_{24} \sum y_{57} \sum y_{89} \\
& -\sum y_{23} \sum y_{4567}-\sum y_{2345} \sum y_{67}+2 \sum y_{24} \sum y_{6678} \\
& +2 \sum y_{2345} \sum y_{68}-2 \sum y_{234667} .
\end{aligned}
$$

The determination of the other $\left\{n, \delta^{i} \epsilon^{i} \eta^{k}\right\}$ is similar.

The numerical values of the coefficients $\left\{n, \delta^{i} \epsilon^{i} \eta^{k}\right\}$ are given in Table I to 5 decimal places, for $\theta_{0}$ ranging from +0.9 to -1.0 (the interesting range in helicopter theory). In the evaluation of $\left\{n, \delta^{i} \epsilon^{j} \eta^{k}\right\}$ the first $51 y_{m}$ were taken into account. (The accuracy obtainable is thus equivalent to the use of a 101-row approximant to D.) $y_{0}$ to $y_{20}$ were computed in some instances to 6 , in some instances to 7 decimal places; $y_{21}$ to $y_{50}$ were computed to 7 decimal places. It is expected that the entries of Table $\mathrm{I}$ are in error by not more than 2 units in the fif th decimal place. ${ }^{5}$

It is readily seen that the present method is not limited to Eq. (1), but can be extended to the general Hill differential equation where $\theta_{m} e^{i m} \psi$ form a convergent series. that

For the special case of Mathieu's equation $(\epsilon=\eta=0)$, one finds by (23) and (27)

$$
\begin{aligned}
\mathcal{D} & =D_{1}\left(D_{0}-\delta y_{01} D_{2}\right)=1-2 \delta \sum_{k=0}^{\infty} \frac{1}{\theta_{0}-k^{2}} \cdot \frac{1}{\theta_{0}-(k+1)^{2}}+O\left(\delta^{2}\right) \\
& =1-2 \delta \frac{\pi \cot \pi \sqrt{\theta_{0}}}{\left(4 \theta_{0}-1\right) \sqrt{\theta_{0}}}+O\left(\delta^{2}\right) .
\end{aligned}
$$

5 An experienced computer can calculate a column of Table I in somewhat less than a day. 
This formula was used by $\mathrm{H}$. Bremekamp in 1926 in a study of the flow of electrons in metals. ${ }^{6}$

4. Example (a). Given $\theta=0.2, \theta_{1}=0.19685+0.33465 i, \theta_{2}=0.03875-0.10258 i$. Determine $v_{1}, v_{2}$. One finds $\delta=0.15074, \epsilon=0.01202, \eta=-0.01635$, and, by Table I, $D_{0}=1.8422, D_{1}=0.9434, D_{2}=0.9934, D_{3}=0.9980, D_{4}=0.999, D_{6}=D_{6}=1.000$. Likewise, by Table II, $\mathcal{D}=2.3291$. Therefore, $q=\sqrt{\mathscr{D}} \sin \pi \sqrt{\theta_{0}}=1.5052$ and by (13b) $\sigma_{1}=0.30782+i / 2, \sigma_{2}=-0.30782-i / 2$. The associated functions $v_{1}(\psi)$ and $v_{2}(\psi)$ are determined from the equation system (9). Normalizing to $c_{0}=1$, and using the equations $k=-4$ to $k=+4$, one obtains ${ }^{7}$

$$
\begin{aligned}
v_{1}= & (-0.1898+1.9817 i) e^{+0.3078 \psi}\left\{-0.0958 \cos \frac{\psi}{2}+\sin \frac{\psi}{2}+0.2076 \cos \frac{3 \psi}{2}\right. \\
& -0.0083 \sin \frac{3 \psi}{2}-0.0125 \cos \frac{5 \psi}{2}-0.0038 \sin \frac{5 \psi}{2}+0.0008 \cos \frac{7 \psi}{2} \\
& \left.+0.0019 \sin \frac{7 \psi}{2}+\cdots\right\} \\
v_{2}= & (1.6652+0.7467 i) e^{-0.3078 \psi}\left\{\cos \frac{\psi}{2}+0.4484 \sin \frac{\psi}{2}+0.2107 \cos \frac{3 \psi}{2}\right. \\
& +0.0188 \sin \frac{3 \psi}{2}+0.0041 \cos \frac{5 \psi}{2}+0.0101 \sin \frac{5 \psi}{2}+0.0005 \cos \frac{7 \psi}{2} \\
& \left.+0.0020 \sin \frac{7 \psi}{2}+\cdots\right\} .
\end{aligned}
$$

5. Example (b). Given $\theta_{0}=0, \theta_{1}=0.37249+0.63323 i, \theta_{2}=0.13875-0.36728 i$. Determine $\sigma$. One finds

$$
\begin{aligned}
q & =\pi \sqrt{\overline{\mathcal{D} \theta_{0}}} \cdot \sin \pi \sqrt{\theta_{0}} / \pi \sqrt{\theta_{0}}=\pi \sqrt{0.4392}=2.082, \\
\sigma_{1} & =-\sigma_{2}=0.4339+i / 2 .
\end{aligned}
$$

- M. J. O. Strutt, loc. cit., p. 26.

${ }^{7}$ Note that the use of $\sigma_{1}=0.30782-i / 2$ leads to the above expression of $v_{1}$ when $c_{1}$ is normalized to 1 , and to the conjugate complex of the above when $c_{0}$ is normalized to 1 . 
TABLE I.* Numerical values of $\left\{n, \delta^{i} e^{j} \eta^{k}\right\}$.

\begin{tabular}{|c|c|c|c|c|c|c|c|c|}
\hline$\theta_{0}$ & .9 & .8 & .7 & .6 & .5 & .45 & .4 & .35 \\
\hline$\left\{\begin{array}{ll}0, & \delta\} \\
0, & \in\} \\
0, & \eta\} \\
0, & \left.\delta^{2}\right\} \\
0, & \delta \in\} \\
0, & \delta \eta\} \\
0, & \left.\delta^{2}\right\} \\
0, & \left.e^{2}\right\} \\
0, & \ln \} \\
0, & \eta^{2} \\
0, & \delta^{2} \\
0, & \left.\delta^{2} \eta\right\} \\
0, & \left.\delta e^{2}\right\} \\
0, & \delta \in \eta\}\end{array}\right\}$ & $\begin{array}{r}7.83180 \\
-.90665 \\
6.36577 \\
-.55015 \\
.52473 \\
-.15952 \\
.00265 \\
-.41427 \\
-.00590 \\
-.00638 \\
-.00463 \\
.00049 \\
.00317 \\
-.00021\end{array}$ & $\begin{array}{r}4.63590 \\
-.24871 \\
3.51924 \\
-.30117 \\
.27858 \\
-.08611 \\
.00143 \\
-.22658 \\
-.00363 \\
-.00342 \\
-.00248 \\
.00025 \\
.00170 \\
-.00012\end{array}$ & $\begin{array}{r}3.70199 \\
.00257 \\
2.63695 \\
-.22352 \\
.20047 \\
-.06307 \\
.00104 \\
-.16801 \\
-.00301 \\
-.00249 \\
-.00178 \\
.00019 \\
.00123 \\
-.00009\end{array}$ & $\begin{array}{r}3.38325 \\
.16467 \\
2.27050 \\
-.19037 \\
.16578 \\
-.05310 \\
.00087 \\
-.14320 \\
-.00278 \\
-.00208 \\
-.00150 \\
.00015 \\
.00103 \\
-.00008\end{array}$ & $\begin{array}{r}3.38203 \\
.30899 \\
2.14607 \\
-.17858 \\
.15052 \\
-.04908 \\
.00081 \\
-.13403 \\
-.00280 \\
-.00191 \\
-.00136 \\
.00014 \\
.00095 \\
-.00007\end{array}$ & $\begin{array}{r}3.48245 \\
.38655 \\
2.15140 \\
-.17822 \\
.14789 \\
-.04867 \\
.00080 \\
-.13372 \\
-.00292 \\
-.00189 \\
-.00133 \\
.00014 \\
.00093 \\
-.00007\end{array}$ & $\begin{array}{r}3.65865 \\
.47423 \\
2.20218 \\
-.18162 \\
.14837 \\
-.04928 \\
.00081 \\
-.13622 \\
-.00306 \\
-.00190 \\
-.00133 \\
.00013 \\
.00094 \\
-.00007\end{array}$ & $\begin{array}{r}3.92977 \\
.57886 \\
2.30622 \\
-.18937 \\
.15229 \\
-.05105 \\
.00083 \\
-.14199 \\
-.00330 \\
-.00196 \\
-.00136 \\
.00014 \\
.00096 \\
-.00007\end{array}$ \\
\hline$\left\{\begin{array}{l}1,8\} \\
1, \\
1, \eta\} \\
1, \delta^{2} \\
1, \delta \in\} \\
1, \delta \eta \\
\left.1, \delta^{2}\right\} \\
\left.1, e^{2}\right\} \\
1, e \eta\} \\
1, \eta^{2}\end{array}\right\}$ & $\begin{array}{r}-3.27931 \\
-1.26507 \\
-.80268 \\
-04439 \\
-.01646 \\
.00739 \\
-.00009 \\
.03150 \\
.00132 \\
.00019\end{array}$ & $\begin{array}{r}-1.61410 \\
-.63934 \\
-.38700 \\
.02131 \\
-.00763 \\
.00351 \\
-.00004 \\
.01513 \\
.00063 \\
.00009\end{array}$ & $\begin{array}{r}-1.05991 \\
-.43033 \\
-.24905 \\
.01366 \\
-.00471 \\
.00223 \\
-.00003 \\
.00970 \\
.00041 \\
.00006\end{array}$ & $\begin{array}{r}-.78342 \\
-.32553 \\
-.18048 \\
.00986 \\
-.00328 \\
.00160 \\
-.00002 \\
.00701 \\
.00030 \\
.00004\end{array}$ & $\begin{array}{r}-.61797 \\
-.26241 \\
-.13964 \\
.00759 \\
-.00243 \\
.00122 \\
-.00002 \\
.00540 \\
.00024 \\
.00002\end{array}$ & $\begin{array}{r}-.55795 \\
-.23943 \\
-.12488 \\
.00678 \\
-.00213 \\
.00109 \\
-.00001 \\
.00482 \\
.00020 \\
.00002\end{array}$ & $\begin{array}{r}-.50802 \\
-.22021 \\
-.11264 \\
.00610 \\
-.00187 \\
.00098 \\
-.00001 \\
.00434 \\
.00019 \\
.00002\end{array}$ & $\begin{array}{r}-.46584 \\
-.20392 \\
-.10233 \\
.00553 \\
-.00166 \\
.00088 \\
-.00001 \\
.00394 \\
.00017 \\
.00002\end{array}$ \\
\hline$\left\{\begin{array}{ll}2, & \delta \\
2, & 6 \\
2, & 7 \\
2, & 8 \\
2, & 8 \\
2, & 8 \\
2, & \epsilon^{2}\end{array}\right\}$ & $\begin{array}{r}-.05351 \\
-.03050 \\
-.00619 \\
.00025 \\
.00001 \\
.00002 \\
.00017\end{array}$ & $\begin{array}{r}-.05160 \\
-.02958 \\
-.00591 \\
.00024 \\
.00001 \\
.00002 \\
.00017\end{array}$ & $\begin{array}{r}-.04981 \\
-.02872 \\
-.00565 \\
.00023 \\
.00001 \\
.00002 \\
.00016\end{array}$ & $\begin{array}{r}-.04813 \\
-.02791 \\
-.00541 \\
.00021 \\
.00001 \\
.00002 \\
.00015\end{array}$ & $\begin{array}{r}-.04654 \\
-.02714 \\
-.00519 \\
.00021 \\
.00001 \\
.00002 \\
.00015\end{array}$ & $\begin{array}{r}-.04579 \\
-.02678 \\
-.00508 \\
.00020 \\
.00001 \\
.00002 \\
.00014\end{array}$ & $\begin{array}{r}-.04505 \\
-.02642 \\
-.00498 \\
.00020 \\
.00002 \\
.00002 \\
.00014\end{array}$ & $\begin{array}{r}-.04434 \\
-.02607 \\
-.00487 \\
.00019 \\
.00002 \\
.00002 \\
.00013\end{array}$ \\
\hline$\left\{\begin{array}{l}3,8\} \\
3, e\} \\
3, y\} \\
3, \delta^{2} \\
3, \delta^{2} \\
\left.3, e^{2}\right\}\end{array}\right\}$ & $\begin{array}{r}-.01368 \\
-.00914 \\
-.00092 \\
.00003 \\
.00001 \\
.00002\end{array}$ & $\begin{array}{r}-.01349 \\
-.00903 \\
-.00090 \\
.00003 \\
.00001 \\
.00002\end{array}$ & $\begin{array}{r}-.01330 \\
-.00892 \\
-.00088 \\
.00003 \\
.00001 \\
.00002\end{array}$ & $\begin{array}{r}-.01311 \\
-.00881 \\
-.00086 \\
.00003 \\
.00001 \\
.00002\end{array}$ & $\begin{array}{r}-.01293 \\
-.00871 \\
-.00086 \\
.00003 \\
.00001 \\
.00002\end{array}$ & $\begin{array}{r}-.01284 \\
-.00866 \\
-.00084 \\
.00003 \\
.00001 \\
.00002\end{array}$ & $\begin{array}{r}-.01275 \\
-.00861 \\
-.00084 \\
.00003 \\
.00001 \\
.00002\end{array}$ & $\begin{array}{r}-.01267 \\
-.00856 \\
-.00083 \\
.00003 \\
.00001 \\
.00002\end{array}$ \\
\hline$\left\{\begin{array}{l}4,8\} \\
4,6\} \\
4,7\} \\
4,8 \in\}\end{array}\right.$ & $\begin{array}{r}-.00551 \\
-.00401 \\
-.00024 \\
.00001\end{array}$ & $\begin{array}{r}-.00546 \\
-.00399 \\
-.00024 \\
.00001\end{array}$ & $\begin{array}{r}-.00543 \\
-.00396 \\
-.00023 \\
.00001\end{array}$ & $\begin{array}{r}-.00538 \\
-.00393 \\
-.00023 \\
.00001\end{array}$ & $\begin{array}{r}-.00534 \\
-.00391 \\
-.00023 \\
.00001\end{array}$ & $\begin{array}{r}-.00532 \\
-.00390 \\
-.00023 \\
.00001\end{array}$ & $\begin{array}{r}-.00530 \\
-.00388 \\
-.00023 \\
.00001\end{array}$ & $\begin{array}{r}-.00528 \\
-.00387 \\
-.00023 \\
.00001\end{array}$ \\
\hline$\left\{\begin{array}{l}5,8\} \\
5,5\} \\
5,2\}\end{array}\right.$ & $\begin{array}{r}-.00276 \\
-.00213 \\
-.00008\end{array}$ & $\begin{array}{r}-.00275 \\
-.00212 \\
-.00008\end{array}$ & $\begin{array}{r}-.00273 \\
-.00211 \\
-.00008\end{array}$ & $\begin{array}{l}-.00272 \\
-.00210 \\
-.00008\end{array}$ & $\begin{array}{r}-.00271 \\
-.00209 \\
-.00008\end{array}$ & $\begin{array}{r}-.00270 \\
-.00209 \\
-.00008\end{array}$ & $\begin{array}{r}-.00269 \\
-.00208 \\
-.00008\end{array}$ & $\begin{array}{r}-.00269 \\
-.00208 \\
-.00008\end{array}$ \\
\hline$\left\{\begin{array}{ll}6, & 8 \\
6, & 1\end{array}\right\}$ & $\begin{array}{r}-.00158 \\
-.00126 \\
-.00003\end{array}$ & $\begin{array}{r}-.00157 \\
-.00126 \\
-.00003\end{array}$ & $\begin{array}{r}-.00157 \\
-.00126 \\
-.00003\end{array}$ & $\begin{array}{l}-.00156 \\
-.00125 \\
-.00003\end{array}$ & $\begin{array}{r}-.00155 \\
-.00125 \\
-.00003\end{array}$ & $\begin{array}{r}-.00155 \\
-.00125 \\
-.00003\end{array}$ & $\begin{array}{r}-.00155 \\
-.00125 \\
-.00003\end{array}$ & $\begin{array}{l}-.00155 \\
-.00125 \\
-.00003\end{array}$ \\
\hline$\theta_{0}$ & .3 & .25 & .2 & .15 & & & .05 & $0 *$ \\
\hline 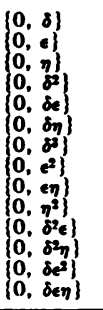 & $\begin{array}{r}4.33216 \\
.71097 \\
2.48046 \\
-.20279 \\
.16054 \\
-.05432 \\
.00089 \\
-.15201 \\
-.00364 \\
-.00208 \\
-.00144 \\
.00014 \\
.00103 \\
-.00007\end{array}$ & $\begin{array}{r}4.93480 \\
.88889 \\
2.75850 \\
-.22456 \\
.17498 \\
-.05977 \\
.00098 \\
-.16827 \\
-.00416 \\
-.00228 \\
-.00158 \\
.00015 \\
.00112 \\
-.00007\end{array}$ & $\begin{array}{r}5.87874 \\
1.14867 \\
3.21013 \\
-.26021 \\
.19957 \\
-.06883 \\
.00112 \\
-.19494 \\
-.00496 \\
-.00262 \\
-.00180 \\
.00018 \\
.00129 \\
-.00008\end{array}$ & $\begin{array}{r}7.49588 \\
1.57391 \\
4.00081 \\
-.32296 \\
.24378 \\
-.08490 \\
.00138 \\
-.24186 \\
-.00630 \\
-.00322 \\
-.00221 \\
.00022 \\
.00160 \\
-.00009\end{array}$ & & $\begin{array}{l}78515 \\
41481 \\
62957 \\
45255 \\
33621 \\
11824 \\
00192 \\
33884 \\
00905 \\
00448 \\
00305 \\
00032 \\
00222 \\
00012\end{array}$ & $\begin{array}{r}20.74569 \\
4.92154 \\
10.59568 \\
-.84825 \\
.02017 \\
-.22028 \\
.00357 \\
-.63496 \\
-.01736 \\
-00832 \\
-.00566 \\
.00059 \\
.00412 \\
-.00023\end{array}$ & $\begin{array}{r}1.000000 \\
.250000 \\
.500000 \\
-.039865 \\
.028683 \\
-.010290 \\
.000166 \\
-.029835 \\
-.000836 \\
-.000388 \\
-.000262 \\
.000028 \\
.000194 \\
-.000012\end{array}$ \\
\hline$\left\{\begin{array}{l}1, \delta\} \\
1, c\} \\
1, \eta \\
1, \delta^{2} \\
1, \delta \in \\
1, \delta \eta \\
1, \delta^{3} \\
1, c^{2} \\
1,-\eta \\
1, \eta^{3}\end{array}\right\}$ & $\begin{array}{r}-.42975 \\
-.18993 \\
-.09354 \\
.00505 \\
-.00149 \\
.00080 \\
-.00001 \\
.00359 \\
.00014 \\
.00002\end{array}$ & $\begin{array}{r}-.39853 \\
-.17778 \\
-.08595 \\
.00463 \\
-.00134 \\
.00073 \\
-.00001 \\
.00330 \\
.00014 \\
.00002\end{array}$ & $\begin{array}{r}-.37126 \\
-.16712 \\
-.07934 \\
.00426 \\
-.00120 \\
.00067 \\
-.00001 \\
.00303 \\
.00012 \\
.00001\end{array}$ & $\begin{array}{r}-.34726 \\
-.15769 \\
-.07355 \\
.00395 \\
-.00109 \\
.00062 \\
-.00001 \\
.00281 \\
.00012 \\
.00001\end{array}$ & & $\begin{array}{l}32596 \\
14929 \\
.06844 \\
00366 \\
00099 \\
00057 \\
00001 \\
.00261 \\
00011 \\
.00001\end{array}$ & $\begin{array}{r}-.30694 \\
-.14176 \\
-.06388 \\
.00341 \\
-.00091 \\
.00053 \\
-.00001 \\
.00243 \\
.00011 \\
.00001\end{array}$ & $\begin{array}{r}-.28987 \\
-.13496 \\
-.05980 \\
.00319 \\
-.00083 \\
.00050 \\
-.00001 \\
.00227 \\
.00010 \\
.00001\end{array}$ \\
\hline
\end{tabular}

* Note 1. $\left\{n, \delta^{i} e^{j} \eta^{k}\right\}$ which are less than .00001 in magnitude, or for which $n>6$, are not shown.

* Note 2. For $\theta_{0}=0\left(y_{0}=\infty\right)$ the coefficients $\frac{A_{\delta}^{0}}{y_{0}}, \frac{A_{E}^{0}}{y_{0}}, \cdots$ of $\frac{D_{0}}{y_{0}}$ are given. 
TABLE I. (Continued)

\begin{tabular}{|c|c|c|c|c|c|c|c|c|}
\hline$\theta$ & .3 & .25 & .2 & \multicolumn{2}{|c|}{.15} & 1 & .05 & $0 * *$ \\
\hline$\left\{\begin{array}{l}2, \delta\} \\
2, c\} \\
2, y\} \\
2, \delta 2 \\
2, \delta \in\} \\
2, \delta \eta\} \\
\left.2, e^{2}\right\}\end{array}\right\}$ & $\begin{array}{r}-.04365 \\
-.02573 \\
-.00477 \\
.00019 \\
.00002 \\
.00002 \\
.00013\end{array}$ & $\begin{array}{r}-.04297 \\
-.02540 \\
-.00468 \\
.00018 \\
.00002 \\
.00002 \\
.00013\end{array}$ & $\begin{array}{r}-.04232 \\
-.02507 \\
-.00458 \\
.00018 \\
.00002 \\
.00002 \\
.00013\end{array}$ & \multicolumn{2}{|c|}{$\begin{array}{r}-.04168 \\
-.02475 \\
-.00450 \\
.00018 \\
.00002 \\
.00002 \\
.00013\end{array}$} & $\begin{array}{l}4106 \\
2445 \\
0442 \\
0017 \\
0002 \\
0002 \\
0012\end{array}$ & $\begin{array}{r}-.04045 \\
-.02415 \\
-.00433 \\
.00017 \\
.00002 \\
.00002 \\
.00012\end{array}$ & $\begin{array}{r}-.03987 \\
-.02385 \\
-.00425 \\
.00017 \\
.00002 \\
.00002 \\
.00012\end{array}$ \\
\hline$\left\{\begin{array}{ll}3, & 8 \\
3, & 1 \\
3, & 7 \\
3, & 8^{2} \\
3, & 8 \\
3, & \left.e^{2}\right\}\end{array}\right\}$ & $\begin{array}{r}-.01258 \\
-.00851 \\
-.00082 \\
.00003 \\
.00001 \\
.00002\end{array}$ & $\begin{array}{r}-.01250 \\
-.00846 \\
-.00081 \\
.00002 \\
.00001 \\
.00002\end{array}$ & $\begin{array}{r}-.01241 \\
-.00842 \\
-.00080 \\
.00002 \\
.00001 \\
.00002\end{array}$ & \multicolumn{2}{|c|}{$\begin{array}{r}-.01233 \\
-.00837 \\
-.00079 \\
.00002 \\
.00001 \\
.00002 \\
\end{array}$} & $\begin{array}{r}-.01225 \\
-.00832 \\
-.00078 \\
.00002 \\
.00001 \\
.00002 \\
\end{array}$ & $\begin{array}{r}-.01217 \\
-.00827 \\
-.00078 \\
.00002 \\
.00001 \\
.00002\end{array}$ & $\begin{array}{r}-.01209 \\
-.00823 \\
-.00077 \\
.00002 \\
.00001 \\
.00002\end{array}$ \\
\hline$\left\{\begin{array}{l}4,8 \\
4,6 \\
4,7\end{array}\right\}$ & $\begin{array}{r}-.00526 \\
-.00386 \\
-.00022\end{array}$ & $\begin{array}{r}-.00524 \\
-.00385 \\
-.00022\end{array}$ & $\begin{array}{r}-.00522 \\
-.00383 \\
-.00022\end{array}$ & \multicolumn{2}{|c|}{$\begin{array}{r}-.00520 \\
-.00382 \\
-.00022 \\
\end{array}$} & $\begin{array}{r}-.00518 \\
-.00381 \\
-.00022 \\
\end{array}$ & $\begin{array}{r}-.00516 \\
-.00380 \\
-.00022\end{array}$ & $\begin{array}{r}-.00514 \\
-.00378 \\
-.00022\end{array}$ \\
\hline$\left\{\begin{array}{l}\{5,8\} \\
\{5,1) \\
\{5,7\}\end{array}\right.$ & $\begin{array}{l}-.00268 \\
-.00207 \\
-.00008\end{array}$ & $\begin{array}{r}-.00268 \\
-.00207 \\
-.00008\end{array}$ & $\begin{array}{r}-.00267 \\
-.00207 \\
-.00008\end{array}$ & \multicolumn{2}{|c|}{$\begin{array}{r}-.00266 \\
-.00206 \\
-.00008 \\
\end{array}$} & $\begin{array}{r}-.00266 \\
-.00206 \\
-.00008 \\
\end{array}$ & $\begin{array}{l}-.00265 \\
-.00205 \\
-.00008\end{array}$ & $\begin{array}{r}-.00264 \\
-.00205 \\
-.00008\end{array}$ \\
\hline$\left\{\begin{array}{l}6,8\} \\
6, \cdot 1\} \\
6,7\}\end{array}\right.$ & $\begin{array}{r}-.00155 \\
-.00124 \\
-.00003\end{array}$ & $\begin{array}{r}-.00155 \\
-.00124 \\
-.00003\end{array}$ & $\begin{array}{r}-.00154 \\
-.00124 \\
-.00003\end{array}$ & \multicolumn{2}{|c|}{$\begin{array}{l}-.00154 \\
-.00024 \\
-.00003\end{array}$} & $\begin{array}{r}-.00154 \\
-.00124 \\
-.00003\end{array}$ & $\begin{array}{r}-.00153 \\
-.00123 \\
-.00003\end{array}$ & $\begin{array}{r}-.00153 \\
-.00123 \\
-.00003\end{array}$ \\
\hline 0. & -.05 & -.1 & -.15 & -.2 & -.25 & -.3 & -.35 & -.4 \\
\hline 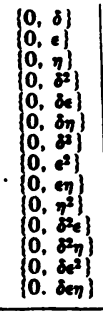 & $\begin{array}{r}-19.32207 \\
-5.06707 \\
-9.46238 \\
.75141 \\
-.53194 \\
.19281 \\
-.00311 \\
.56222 \\
.01609 \\
.00724 \\
.00488 \\
-.00048 \\
-.00357 \\
.00023\end{array}$ & $\begin{array}{r}-9.35137 \\
-2.56220 \\
-4.48740 \\
.35491 \\
-.24724 \\
.09051 \\
-.00146 \\
.26550 \\
.00775 \\
.00338 \\
.00227 \\
-.00023 \\
-.00167 \\
.00010\end{array}$ & $\begin{array}{r}-6.04482 \\
-1.72447 \\
-2.84360 \\
.22402 \\
-.15352 \\
.05679 \\
-.00092 \\
.16755 \\
.00500 \\
.00211 \\
.00141 \\
-.00014 \\
-.00103 \\
.00006\end{array}$ & $\begin{array}{r}-4.40273 \\
-1.30380 \\
-2.03119 \\
.15940 \\
-.10745 \\
.04017 \\
-.00065 \\
.11919 \\
.00363 \\
.00149 \\
.00098 \\
-.00010 \\
-.00073 \\
.00004\end{array}$ & $\begin{array}{r}-3.42538 \\
-1.05015 \\
-1.55045 \\
.12120 \\
-.08037 \\
.03036 \\
-.00048 \\
.09061 \\
.00281 \\
.00112 \\
.00074 \\
-.00008 \\
-.00055 \\
.00003\end{array}$ & $\begin{array}{r}-2.77963 \\
-.88013 \\
-1.23488 \\
.09616 \\
-.06274 \\
.02396 \\
-.00038 \\
.07188 \\
.00226 \\
.00088 \\
.00059 \\
-.00006 \\
-.00044 \\
.00003\end{array}$ & $\begin{array}{r}-2.32283 \\
-.75802 \\
-1.01322 \\
.07860 \\
-.05042 \\
.01947 \\
-.00031 \\
.05874 \\
.00188 \\
.00072 \\
.00047 \\
-.00006 \\
-.00035 \\
.00002\end{array}$ & $\begin{array}{r}-1.98371 \\
-.06590 \\
-.84989 \\
.06569 \\
-.04144 \\
.01617 \\
-.00026 \\
.04908 \\
.00160 \\
.00059 \\
.00039 \\
-.00004 \\
-.00029 \\
.00001\end{array}$ \\
\hline$\left\{\begin{array}{l}1,8\} \\
1,0\} \\
1, \eta\} \\
1,8^{2} \\
1,8 \\
1,8 \eta \\
1,8\} \\
1, e^{2} \\
1,0 \eta \\
1, \eta^{2}\end{array}\right\}$ & $\begin{array}{r}-.27445 \\
-.12880 \\
-.05614 \\
.00299 \\
-.00076 \\
.00046 \\
-.00001 \\
.00213 \\
.00009 \\
.00001\end{array}$ & $\begin{array}{r}-.26046 \\
-.12318 \\
-.05282 \\
.00281 \\
-.00069 \\
.00043 \\
-.00001 \\
.00200 \\
.00009 \\
.00001\end{array}$ & $\begin{array}{r}-.24772 \\
-.11805 \\
-.04981 \\
.00264 \\
-.00064 \\
.00040 \\
-.00001 \\
.00188 \\
.00008 \\
.00001\end{array}$ & $\begin{array}{r}-.23607 \\
-.11332 \\
-.04707 \\
.00249 \\
-.00059 \\
.00038 \\
-.00001 \\
.00178 \\
.00008 \\
.00001\end{array}$ & $\begin{array}{r}-.22538 \\
-.10897 \\
-.04457 \\
.00235 \\
-.00054 \\
.00036 \\
.00168 \\
.00007 \\
.00001\end{array}$ & $\begin{array}{r}-.21553 \\
-.10494 \\
-.04227 \\
.00223 \\
-.00050 \\
.00034 \\
.00159 \\
.00007 \\
.00001\end{array}$ & $\begin{array}{r}-.20643 \\
-.10120 \\
-.04016 \\
.00211 \\
-.00047 \\
.00032 \\
.00151 \\
.00007 \\
.00001\end{array}$ & $\begin{array}{r}-.19800 \\
-.09772 \\
-.03821 \\
.00201 \\
-.00043 \\
.00030 \\
.00143 \\
.00006\end{array}$ \\
\hline$\left\{\begin{array}{l}2, \delta\} \\
2, e\} \\
2, \eta\} \\
\left.2, \delta^{2}\right\} \\
2,8]\} \\
2, \delta \eta\} \\
\left.2, e^{2}\right\}\end{array}\right\}$ & $\begin{array}{r}-.03929 \\
-.02357 \\
-.00417 \\
.00016 \\
.00002 \\
.00002 \\
.00011\end{array}$ & $\begin{array}{r}-.03873 \\
-.02328 \\
-.00409 \\
.00016 \\
.00002 \\
.00002 \\
.00011\end{array}$ & $\begin{array}{r}-.03819 \\
-.02301 \\
-.00401 \\
.00016 \\
.00002 \\
.00001 \\
.00011\end{array}$ & $\begin{array}{r}-.03766 \\
-.02275 \\
-.00394 \\
.00015 \\
.00002 \\
.00001 \\
.00011\end{array}$ & $\begin{array}{r}-.03714 \\
-.02249 \\
-.00387 \\
.00015 \\
.00002 \\
.00001 \\
.00011\end{array}$ & $\begin{array}{r}-.03663 \\
-.02223 \\
-.00380 \\
.00015 \\
.00002 \\
.00001 \\
.00010\end{array}$ & $\begin{array}{r}-.03614 \\
-.02198 \\
-.00373 \\
.00015 \\
.00002 \\
.00001 \\
.00010\end{array}$ & $\begin{array}{r}-.03566 \\
-.02174 \\
-.00367 \\
.00014 \\
.00002 \\
.00001 \\
.00010\end{array}$ \\
\hline$\left\{\begin{array}{l}3, \delta\} \\
3,0\} \\
3, \eta\} \\
3, \delta^{2} \\
3, \delta^{2} \\
\left.3, e^{2}\right\}\end{array}\right\}$ & $\begin{array}{r}-.01201 \\
-.00818 \\
-.00077 \\
.00002 \\
.00001 \\
.00002\end{array}$ & $\begin{array}{r}-.01193 \\
-.00814 \\
-.00076 \\
.00002 \\
.00001 \\
.00002\end{array}$ & $\begin{array}{r}-.01185 \\
-.00809 \\
-.00075 \\
.00002 \\
.00001 \\
.00001\end{array}$ & $\begin{array}{r}-.01178 \\
-.00805 \\
-.00075 \\
.00002 \\
.00001 \\
.00001\end{array}$ & $\begin{array}{r}-.01170 \\
-.00801 \\
-.00074 \\
.00002 \\
.00001 \\
.00001\end{array}$ & $\begin{array}{r}-.01163 \\
-.00796 \\
-.00073 \\
.00002 \\
.00001 \\
.00001\end{array}$ & $\begin{array}{r}-.01155 \\
-.00792 \\
-.00073 \\
.00002 \\
.00001 \\
.00001\end{array}$ & $\begin{array}{r}-.01148 \\
-.00788 \\
-.00072 \\
.00002 \\
.00001 \\
.00001\end{array}$ \\
\hline$\left\{\begin{array}{l}4,8\} \\
4,1\} \\
4,7\}\end{array}\right.$ & $\begin{array}{r}-.00512 \\
-.00377 \\
-.00022\end{array}$ & $\begin{array}{r}-.00510 \\
-.00376 \\
-.00022\end{array}$ & $\begin{array}{r}-.00509 \\
-.00375 \\
-.00021\end{array}$ & $\begin{array}{r}-.00507 \\
-.00374 \\
-.00021\end{array}$ & $\begin{array}{r}-.00505 \\
-.00372 \\
-.00021\end{array}$ & $\begin{array}{l}-.00503 \\
-.00371 \\
-.00021\end{array}$ & $\begin{array}{r}-.00501 \\
-.00370 \\
-.00021\end{array}$ & $\begin{array}{r}-.00500 \\
-.00369 \\
-.00021\end{array}$ \\
\hline$\left\{\begin{array}{l}\{5,8\} \\
\{5,1\} \\
5,7\}\end{array}\right.$ & $\begin{array}{r}-.00264 \\
-.00204 \\
-.00008\end{array}$ & $\begin{array}{l}-.00263 \\
-.00204 \\
-.00008\end{array}$ & $\begin{array}{r}-.00262 \\
-.00204 \\
-.00008\end{array}$ & $\begin{array}{l}-.00262 \\
-.00203 \\
-.00008\end{array}$ & $\begin{array}{l}-.00261 \\
-.00203 \\
-.00008\end{array}$ & $\begin{array}{l}-.00261 \\
-.00202 \\
-.00008\end{array}$ & $\begin{array}{r}-.00260 \\
-.00202 \\
-.00008\end{array}$ & $\begin{array}{l}-.00259 \\
-.00202 \\
-.00008\end{array}$ \\
\hline$\left\{\begin{array}{l}6,8\} \\
6,8\} \\
6,7\}\end{array}\right.$ & $\begin{array}{r}-.00153 \\
-.00123 \\
-.00003\end{array}$ & $\begin{array}{l}-.00153 \\
-.00123 \\
-.00003\end{array}$ & $\begin{array}{l}-.00152 \\
-.00123 \\
-.00003\end{array}$ & $\begin{array}{l}-.00152 \\
-.00122 \\
-.00003\end{array}$ & $\begin{array}{r}-.00152 \\
-.00122 \\
-.00003\end{array}$ & $\begin{array}{r}-.00152 \\
-.00122 \\
-.00003 \\
\end{array}$ & $\begin{array}{r}-.00151 \\
-.00122 \\
-.00003\end{array}$ & $\begin{array}{l}-.00151 \\
-.00122 \\
-.00003\end{array}$ \\
\hline
\end{tabular}


TABle I. (Continued)

\begin{tabular}{|c|c|c|c|c|c|c|c|c|}
\hline$\theta_{0}$ & -.45 & -.5 & -.55 & -.6 & -.7 & -.8 & -.9 & -1.0 \\
\hline 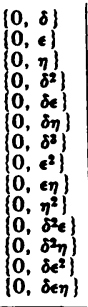 & $\begin{array}{r}-1.72274 \\
-.59385 \\
-.72520 \\
.05584 \\
-.03464 \\
.01368 \\
-.00022 \\
.04172 \\
.00139 \\
.00050 \\
.00033 \\
-.00003 \\
-.00025 \\
.00001\end{array}$ & $\begin{array}{r}-1.51622 \\
-.53588 \\
-.02733 \\
.04813 \\
-.02936 \\
.01172 \\
-.00019 \\
.03595 \\
.00121 \\
.00043 \\
.00028 \\
-.00003 \\
-.00021 \\
.00001\end{array}$ & $\begin{array}{r}-1.34910 \\
-.48819 \\
-.54879 \\
.04195 \\
-.02516 \\
.01016 \\
-.00016 \\
.03133 \\
.00107 \\
.00037 \\
.00024 \\
-.00002 \\
-.00018 \\
.00001\end{array}$ & $\begin{array}{r}-1.21138 \\
-.44823 \\
-.44863 \\
.03691 \\
-.02176 \\
.00888 \\
-.00014 \\
.02757 \\
.00096 \\
.00032 \\
.00021 \\
-.00002 \\
-.00016 \\
.00001\end{array}$ & $\begin{array}{r}-.99849 \\
-.38497 \\
-.38670 \\
.02924 \\
-.01666 \\
.00695 \\
-.00011 \\
.02184 \\
.00078 \\
.00025 \\
.00016 \\
-.00001 \\
-.00012\end{array}$ & $\begin{array}{r}-.84237 \\
-.33706 \\
-.31617 \\
.02374 \\
-.01306 \\
.00559 \\
-.00009 \\
.01772 \\
.00064 \\
.00020 \\
.00013 \\
-.00001 \\
-.00010\end{array}$ & $\begin{array}{r}-.72362 \\
-.29947 \\
-.26349 \\
.01965 \\
-.01043 \\
.00457 \\
-.00007 \\
.01467 \\
.00055 \\
.00016 \\
.00010 \\
-.00001 \\
-.00008\end{array}$ & $\begin{array}{r}-.63067 \\
-.26917 \\
-.22300 \\
.01652 \\
-.00846 \\
.00380 \\
-.00006 \\
.01233 \\
.00047 \\
.00013 \\
.00008 \\
-.00001 \\
-.00007\end{array}$ \\
\hline$\left\{\begin{array}{l}1, \delta\} \\
1, e\} \\
1, \eta\} \\
1, \delta 2 \\
1, \delta \epsilon\} \\
1, \delta \eta\} \\
\left.1, e^{2}\right\} \\
1, \in \eta\}\end{array}\right\}$ & $\begin{array}{r}-.19017 \\
-.09448 \\
-.03641 \\
.00191 \\
-.00040 \\
.00029 \\
.00136 \\
.00006\end{array}$ & $\begin{array}{r}-.18288 \\
-.09144 \\
-.03473 \\
.00182 \\
-.00037 \\
.00027 \\
.00130 \\
.00006\end{array}$ & $\begin{array}{r}-.17608 \\
-.08859 \\
-.03318 \\
.00173 \\
-.00034 \\
.00026 \\
.00124 \\
.00006\end{array}$ & $\begin{array}{r}-.16971 \\
-.08591 \\
-.03173 \\
.00166 \\
-.00032 \\
.00025 \\
.00118 \\
.00006\end{array}$ & $\begin{array}{r}-.15815 \\
-.08102 \\
-.02911 \\
.00151 \\
-.00028 \\
.00022 \\
.00108 \\
.00005\end{array}$ & $\begin{array}{r}-.14793 \\
-.07664 \\
-.02682 \\
.00139 \\
-.00024 \\
.00020 \\
.00099 \\
.00005\end{array}$ & $\begin{array}{r}-.13882 \\
-.07272 \\
-.02480 \\
.00128 \\
-.00020 \\
.00018 \\
.00092 \\
.00004\end{array}$ & $\begin{array}{r}-.13067 \\
-.06917 \\
-.02300 \\
.00118 \\
-.00018 \\
.00017 \\
.00085 \\
.00003\end{array}$ \\
\hline$\left\{\begin{array}{l}2, \delta\} \\
2, \epsilon\} \\
2, y\} \\
\left.2, \delta^{2}\right\} \\
2, \delta \epsilon\} \\
2, \delta \eta\} \\
\left.2, e^{2}\right\}\end{array}\right\}$ & $\begin{array}{r}-.03519 \\
-.02150 \\
-.00361 \\
.00014 \\
.00002 \\
.00001 \\
.00010\end{array}$ & $\begin{array}{r}-.03473 \\
-.02126 \\
-.00354 \\
.00014 \\
.00002 \\
.00001 \\
.00010\end{array}$ & $\begin{array}{r}-.03428 \\
-.02103 \\
-.00349 \\
.00014 \\
.00002 \\
.00001 \\
.00010\end{array}$ & $\begin{array}{r}-.03384 \\
-.02081 \\
-.00342 \\
.00013 \\
.00002 \\
.00001 \\
.00009\end{array}$ & $\begin{array}{r}-.03300 \\
-.02037 \\
-.00331 \\
.00013 \\
.00002 \\
.00001 \\
.00009\end{array}$ & $\begin{array}{r}-.03219 \\
-.01995 \\
-.00320 \\
.00012 \\
.00002 \\
.00001 \\
.00009\end{array}$ & $\begin{array}{r}-.03141 \\
-.01955 \\
-.00310 \\
.00012 \\
.00002 \\
.00001 \\
.00008\end{array}$ & $\begin{array}{r}-.03067 \\
-.01917 \\
-.00150 \\
.00012 \\
.00002 \\
.00001 \\
.00008\end{array}$ \\
\hline$\left\{\begin{array}{l}3, \delta\} \\
3, \varepsilon\} \\
3, \eta\} \\
\left.3, \delta^{2}\right\} \\
3, \delta \in\} \\
\left.3, e^{2}\right\}\end{array}\right\}$ & $\begin{array}{r}-.01141 \\
-.00784 \\
-.00072 \\
.00002 \\
.00001 \\
.00001\end{array}$ & $\begin{array}{r}-.01134 \\
-.00779 \\
-.00071 \\
.00002 \\
.00001 \\
.00001\end{array}$ & $\begin{array}{r}-.01127 \\
-.00775 \\
-.00071 \\
.00002 \\
.00001 \\
.00001\end{array}$ & $\begin{array}{r}-.01120 \\
-.00771 \\
-.00070 \\
.00002 \\
.00001 \\
.00001\end{array}$ & $\begin{array}{r}-.01106 \\
-.00763 \\
-.00068 \\
.00002 \\
.00001 \\
.00001\end{array}$ & $\begin{array}{r}-.01093 \\
-.00755 \\
-.00067 \\
.00002 \\
.00001 \\
.00001\end{array}$ & $\begin{array}{r}-.01080 \\
-.00748 \\
-.00066 \\
.00002 \\
.00001 \\
.00001\end{array}$ & $\begin{array}{r}-.01067 \\
-.00740 \\
-.00065 \\
.00002 \\
.00001 \\
.00001\end{array}$ \\
\hline$\left\{\begin{array}{l}4,8\} \\
4,6\} \\
4,7\}\end{array}\right\}$ & $\begin{array}{r}-.00498 \\
-.00368 \\
-.00021\end{array}$ & $\begin{array}{l}-.00496 \\
-.00366 \\
-.00020\end{array}$ & $\begin{array}{r}-.00494 \\
-.00365 \\
-.00020\end{array}$ & $\begin{array}{r}-.00492 \\
-.00364 \\
-.00020\end{array}$ & $\begin{array}{r}-.00489 \\
-.00362 \\
-.00020\end{array}$ & $\begin{array}{r}-.00485 \\
-.00360 \\
-.00020\end{array}$ & $\begin{array}{r}-.00482 \\
-.00358 \\
-.00020\end{array}$ & $\begin{array}{r}-.00479 \\
-.00356 \\
-.00020\end{array}$ \\
\hline$\left\{\begin{array}{l}5,8\} \\
5,6\} \\
5,7\}\end{array}\right\}$ & $\begin{array}{l}-.00259 \\
-.00201 \\
-.00008\end{array}$ & $\begin{array}{r}-.00259 \\
-.00201 \\
-.00008\end{array}$ & $\begin{array}{r}-.00258 \\
-.00200 \\
-.00008\end{array}$ & $\begin{array}{r}-.00257 \\
-.00200 \\
-.00008\end{array}$ & $\begin{array}{r}-.00256 \\
-.00199 \\
-.00008\end{array}$ & $\begin{array}{r}-.00255 \\
-.00198 \\
-.00007 \\
\end{array}$ & $\begin{array}{l}-.00253 \\
-.00197 \\
-.00007\end{array}$ & $\begin{array}{r}-.00252 \\
-.00197 \\
-.00007\end{array}$ \\
\hline $\begin{array}{l}\left\{\begin{array}{l}6,8 \\
6,1\end{array}\right\} \\
6,7\}\end{array}$ & $\begin{array}{l}-.00151 \\
-.00122 \\
-.00003\end{array}$ & $\begin{array}{l}-.00151 \\
-.00121 \\
-.00003\end{array}$ & $\begin{array}{r}-.00150 \\
-.00121 \\
-.00003\end{array}$ & $\begin{array}{l}-.00150 \\
-.00121 \\
-.00003\end{array}$ & $\begin{array}{r}-.00150 \\
-.00121 \\
-.00003\end{array}$ & $\begin{array}{r}-.00149 \\
-.00120 \\
-.00003\end{array}$ & $\begin{array}{r}-.00149 \\
-.00120 \\
-.00003\end{array}$ & $\begin{array}{r}-.00149 \\
-.00120 \\
-.00003\end{array}$ \\
\hline
\end{tabular}


TABle II. Expansion of $\mathcal{D}$ (row 1 xrow 2 row 3, see Eq. 23).

Numerical tabulation of the coefficients in row 3 .

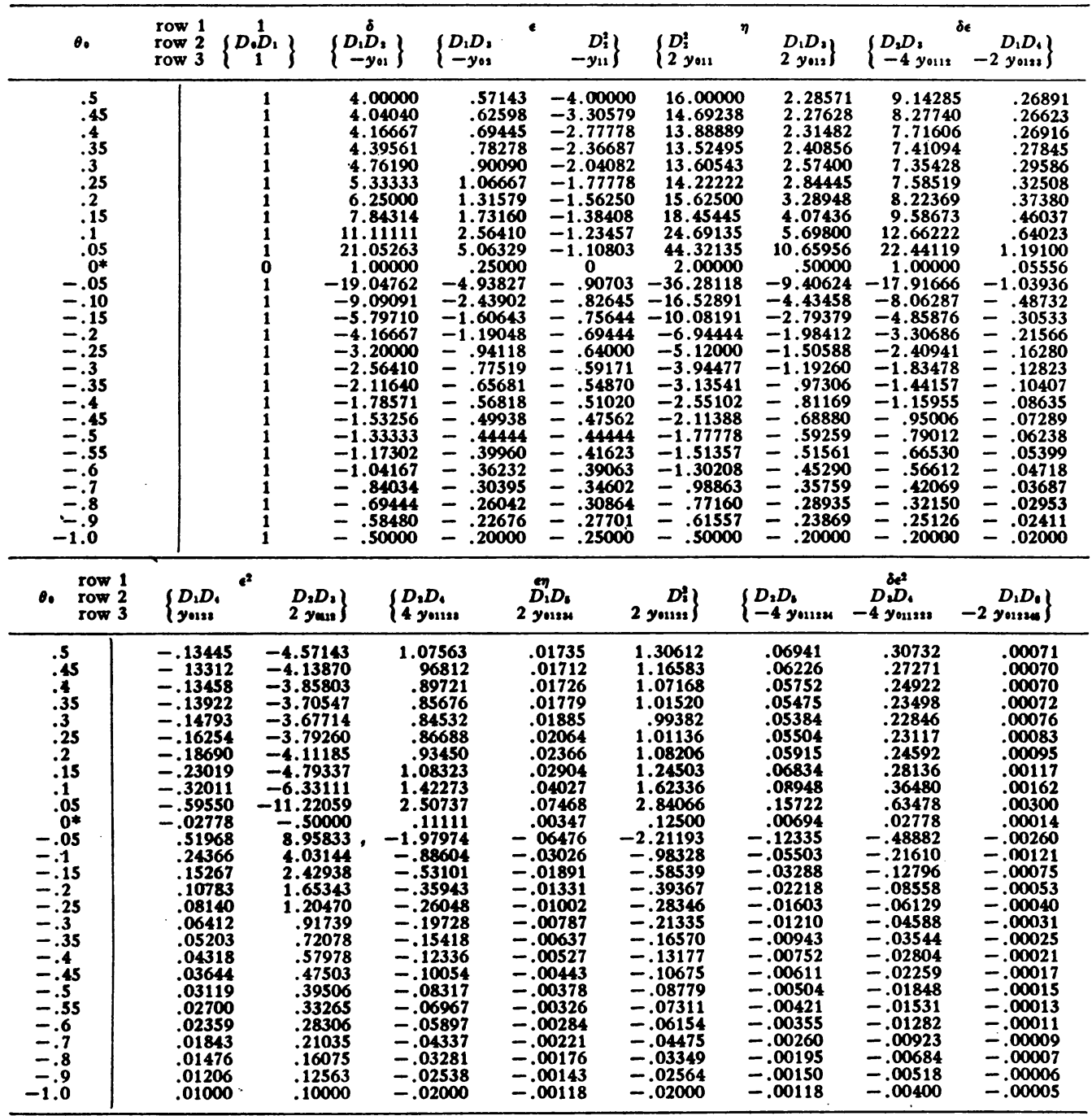

* Note 1. For $\theta_{0}=0 \quad\left(y_{0}=\infty\right)$ the coefficients of $D / y_{0}$ are given. 